\title{
Non-perturbative solutions for lattice quantum gravity
}

\author{
R. Loll \\ Sezione INFN di Firenze, Largo E. Fermi 2, I-50125 Florence, Italy
}

Received 10 February 1995; accepted 11 April 1995

\begin{abstract}
We propose a new, discretized model for the study of $(3+1)$-dimensional canonical quantum gravity, based on the classical $\operatorname{SL}(2, \mathbb{C})$-connection formulation. The discretization takes place on a topological $N^{3}$ lattice with periodic boundary conditions. All operators and wave functions are constructed from one-dimensional link variables, which are regarded as the fundamental building blocks of the theory. The kinematical Hilbert space is spanned by polynomials of certain Wilson loops on the lattice and is manifestly gauge- and diffeomorphism-invariant. The discretized quantum Hamiltonian $\hat{H}$ maps this space into itself. We find a large sector of solutions to the discretized Wheeler-DeWitt equation $\hat{H} \psi=0$, which are labelled by single and multiple Polyakov loops. These states have a finite norm with respect to a natural scalar product on the space of holomorphic $\operatorname{SL}(2, \mathbb{C})$-Wilson loops. We also investigate the existence of further solutions for the case of the $1^{3}$ lattice. Our results provide for the first time a rigorous, regularized framework for studying non-perturbative canonical quantum gravity.
\end{abstract}

\section{Introduction}

All attempts to define a lattice discretization of four-dimensional quantum gravity, in analogy with the rather powerful methods employed in quantum chromodynamics, have been riddled with difficulties. A major problem is that of incorporating the diffeomorphism-invariance of the theory. The discretization typically destroys this symmetry already at the classical level, similar to the way in which a lattice discretization of Yang-Mills theory breaks its rotational symmetry. However, in the case of gravity this is much more serious, since the diffeomorphism group is infinite-dimensional, and acts non-linearly on the underlying manifold. A number of questions arise: (i) does the discretized quantum theory possess a residual diffeomorphism symmetry? (ii) how is the continuum limit to be taken? and (iii) what does the resulting theory look like? 
(This should be a non-perturbative, diffeomorphism-invariant description for quantum gravity in an "unbroken" phase.) The answer to (i) is usually "no", whereas the other two questions are hard to address and have not yet found a satisfactory solution within either Lagrangian or Hamiltonian approaches. Using Euclidean path integral methods, a main problem is that of finding the correct measure, without over- or undercounting the physical configurations (currently the most active research programs are quantum Regge calculus and dynamically triangulated gravity; see Ref. [1] for a recent review). A central problem in the canonical approaches is that of finding a closing algebra of diffeomorphism constraints on the phase space of the regularized theory (see, for example, the discussion in Ref. [2]). The unresolved status of the closure problem is the reason for which many Hamiltonian discretized models have not advanced very far $[3,2]$.

The starting point of this paper is a particular form of Hamiltonian lattice gravity. However, instead of implementing the action of the (three-dimensional) spatial diffeomorphisms directly on the phase space of the theory, we will use a framework in which diffeomorphism invariance is manifest. This approach is inspired by the loop space formulation of canonical quantum gravity, based on the classical reformulation of Einstein gravity in terms of a pair $\left(A_{a}^{i}, \tilde{E}_{i}^{a}\right)$ of $\operatorname{SL}(2, \mathbb{C})$ Yang-Mills variables due to Ashtekar $[4,5]$. In the original paper by Rovelli and Smolin Ref. [6], the wave functions $\psi(\gamma)$ of the quantum theory are labelled by spatial loops $\gamma$, and the diffeomorphism invariance is formally implemented by selecting those wave functions that are invariant when the loop argument $\gamma$ is moved by a diffeomorphism. Physical wave functions therefore depend on diffeomorphism equivalence classes $[\gamma]$ of closed loops. Using these ideas, solutions to all of the quantum constraints, including the Wheeler-DeWitt equation $\hat{H} \psi=0$, have been found [6] (for an overview of this and other solutions, see Ref. [7]). This involves the choice of a regularization and factor ordering for the quantum Hamiltonian constraint $\hat{H}$, and is rather formal, in the sense that there is no well-defined scalar product on the space of solutions, and little control over the influence of different regularizations and factor orderings on the structure of this space. As a result the status of these solutions has remained unclear and the issue of reality conditions could not be addressed. In the Ashtekar formulation in terms of complex canonical variable pairs ( $A_{a}^{i}, \tilde{E}_{i}^{a}$ ), such conditions have to be implemented in the quantum theory to make sure that real, and not complex gravity is described.

Recently, there have been proposals for defining continuum loop representations rigorously, regarding them as non-linear analogues of the Fock representation based on quantum loop states (see Ref. [8] for a review and further references). Central to this line of research are the construction of suitable domains for wave functions depending on connections modulo gauge transformations and diffeomorphism-invariant measures on such spaces. However, so far these efforts have addressed only the kinematical structure of the quantum theory, without incorporating any of the dynamical issues regarding the quantum Hamiltonian. A rigorous analysis of the structure of the solution space to the Wheeler-DeWitt equation and of physical operators on this space is therefore still lacking. In the present work we will suggest a discretized version of canonical quantum gravity that can deal with these issues. 
The discretization will take place on a periodic cubic lattice, but this is not to be thought of as a fixed lattice embedded in physical, Euclidean three-space, but merely as a topological quantity, defined by those of its properties that would remain unaffected by a smooth diffeomorphism. It may thus be thought of as representing a diffeomorphism equivalence class of cubic lattices. All physical quantities are defined in terms of link variables, and the discretized Hamiltonian acts purely combinatorially on wave functions labelled by lattice loops. The formalism is manifestly diffeomorphism- and therefore also scale-invariant. The basic link variables of our formulation are a Kogut-Susskind pair of an $\operatorname{SL}(2, \mathbb{C})$-valued link holonomy and a corresponding $\operatorname{sl}(2, \mathbb{C})$-momentum variable. However, the corresponding basic operators in the quantum representation are not selfadjoint. This is acceptable, since they do not correspond to any physical observables. It comes about since we propose to implement the reality conditions, following the ideas of Refs. [5,3], as a holomorphicity condition on wave functions (this leads to the correct number of degrees of freedom in the quantum theory). Also the Hamiltonian operator turns out to be non-selfadjoint. This is no real reason for concern either, if one accepts the argument that the selfadjointness of the Hamiltonian in a generally covariant theory is not a strict physical requirement (for related discussions, see Ref. [9]).

On a cubic $N^{3}$ lattice with periodic boundary conditions, given a specific discretization of the Hamiltonian $H$ together with a particular operator ordering for $\hat{H}$, we are able to find an infinite set of solutions to the Wheeler-DeWitt equation, that moreover have finite norm with respect to the inner product induced from the original kinematical Hilbert space. They are labelled by so-called Polyakov loops and multiple Polyakov loops, which are well known from their role as order parameters for the phase structure of lattice gauge theory (see, for example, Ref. [10]). Their appearance in the context of lattice gravity is curious, although they play here a quite different role, namely, that of parametrizing the solution space to the Wheeler-DeWitt equation. These solutions are the lattice analogues of the non-intersecting, smooth-loop solutions found by Rovelli and Smolin [6]. This for the first time provides a regularized model for non-perturbative canonical quantum gravity that can be used to study physical observables.

The paper is organized as follows. In the next section we introduce all the necessary ingredients for setting up the Hamiltonian lattice theory, and explain some features of the holomorphic representation we will be using for constructing a scalar product on $\mathrm{SL}(2, \mathbb{C})$ wave functions. We derive an important explicit formula for relating arbitrary polynomials in the $\operatorname{SU}(2)$ and $\operatorname{SL}(2, \mathbb{C})$ theories. In Section 3 we establish a relation between the zero-eigenvalue solutions of our quantum Hamiltonian and another Hamiltonian, induced from the SU(2) theory. This suggests a close connection between the real and the complex, holomorphic theories. Next we demonstrate that there is a large subspace of the Hilbert space that is annihilated by the Hamiltonian constraint. In Section 4 we take a closer look at the special case of the $1^{3}$ lattice and explore the possibility of finding solutions beyond the ones labelled by the Polyakov loops. We illustrate some of the technicalities involved, without being able to identify any solutions explicitly. The last section contains our conclusions and an outlook. 


\section{The general formalism}

We first recall the basic Hamiltonian variables for the SU(2) lattice gauge theory, before discussing the complexified framework for $\operatorname{SL}(2, \mathbb{C})=\operatorname{SU}(2)_{\mathbb{C}}$. This is necessary for setting up a discretized version of the connection formulation of canonical gravity. With each lattice link we associate an element $g \in \mathrm{SU}(2)$, parametrized by a matrix $V(g)$ in the defining two-dimensional representation as

$$
V_{A}^{B}=\left(\begin{array}{cc}
\alpha_{0}+i \alpha_{1} & \alpha_{2}+i \alpha_{3} \\
-\alpha_{2}+i \alpha_{3} & \alpha_{0}-i \alpha_{1}
\end{array}\right)
$$

with $\alpha_{i} \in \mathbb{R}$, and subject to the condition $\sum_{i=0}^{3} \alpha_{i}^{2}=1$. The matrix $V_{A}{ }^{B}$ can be written as a (real) linear combination of the unit matrix $\|$ and the three $\tau$-matrices defined by

$$
\tau_{1}=\left(\begin{array}{cc}
i & 0 \\
0 & -i
\end{array}\right), \quad \tau_{2}=\left(\begin{array}{cc}
0 & 1 \\
-1 & 0
\end{array}\right), \quad \tau_{3}=\left(\begin{array}{cc}
0 & i \\
i & 0
\end{array}\right) .
$$

The $\tau$-matrices satisfy $\left[\tau_{i}, \tau_{j}\right]=2 \epsilon_{i j k} \tau_{k}$. The differential operator

$$
\left(\frac{\partial}{\partial V}\right)_{A}{ }^{B}=\frac{1}{4}\left(\begin{array}{cc}
\partial_{0}-i \partial_{1} & -\partial_{2}-i \partial_{3} \\
\partial_{2}-i \partial_{3} & \partial_{0}+i \partial_{1}
\end{array}\right)
$$

acts on the representation matrices (2.1) and satisfies

$$
\left(\frac{\partial}{\partial V}\right)_{A}^{B} V_{C}^{D}=\frac{1}{2} \delta_{A}^{D} \delta_{C}^{B} .
$$

The operators corresponding to the classical momentum variables $p_{i}$ (with a gauge algebra index $i$ ) are given by

$$
\hat{p}_{i}=-i \tau_{i A}^{B} V_{B}^{C}\left(\frac{\partial}{\partial V}\right)_{C}^{A}
$$

and satisfy

$$
\begin{gathered}
\hat{p}_{i} V_{A}{ }^{C}=-\frac{i}{2} \tau_{i A}^{B} V_{B}{ }^{C}, \\
\hat{p}_{i}\left(V^{-1}\right)_{A}{ }^{C}=\frac{i}{2}\left(V^{-1}\right)_{A}{ }^{B} \tau_{i B}{ }^{C} .
\end{gathered}
$$

In terms of coordinates, we have

$$
\begin{aligned}
& \hat{p}_{1}=\frac{i}{2}\left(\alpha_{1} \partial_{0}-\alpha_{0} \partial_{1}+\alpha_{3} \partial_{2}-\alpha_{2} \partial_{3}\right), \\
& \hat{p}_{2}=\frac{i}{2}\left(\alpha_{2} \partial_{0}-\alpha_{3} \partial_{1}-\alpha_{0} \partial_{2}+\alpha_{1} \partial_{3}\right), \\
& \hat{p}_{3}=\frac{i}{2}\left(\alpha_{3} \partial_{0}+\alpha_{2} \partial_{1}-\alpha_{1} \partial_{2}-\alpha_{0} \partial_{3}\right) .
\end{aligned}
$$

Defining the operator $\hat{V}$ as multiplication by the matrix $V$ therefore leads to the commutation relations 


$$
\begin{aligned}
{\left[\hat{V}_{A}^{B}, \hat{V}_{C}^{D}\right] } & =0, \\
{\left[\hat{p}_{i}, \hat{V}_{A}^{C}\right] } & =-\frac{i}{2} \tau_{i A}{ }^{B} \hat{V}_{B}^{C}, \\
{\left[\hat{p}_{i}, \hat{p}_{j}\right] } & =i \epsilon_{i j k} \hat{p}_{k},
\end{aligned}
$$

which are the quantum equivalents of the Poisson brackets of the corresponding classical quantities. These commutation relations are familiar from the Hamiltonian SU(2)-lattice gauge theory [11], where the $\hat{p}_{i}$ are hermitian operators. Here, in contrast, we shall associate three complex (i.e. $\operatorname{SL}(2, \mathbb{C})$ ) degrees of freedom with each link of the hypercubic lattice. As usual, operators $(\hat{V}, \hat{p})$ associated with different links commute.

The entire construction (2.1)-(2.8) makes sense also if we complexify the group to $\mathrm{SU}(2)_{\mathbb{C}}=\operatorname{SL}(2, \mathbb{C})$. We will denote the corresponding group parameters by complex numbers $\alpha_{i}^{\mathbb{C}}, i=0, \ldots, 3$, again subject to a condition $\sum_{i=0}^{3}\left(\alpha_{i}^{\mathbb{C}}\right)^{2}=1$. The operators $\hat{V}$ and $\hat{p}$ are taken to act on a space of holomorphic wave functions on the group manifold of $\operatorname{SL}(2, \mathbb{C})$, whose inner product will be specified below. Note that due to the non-compactness and non-abelianness of the group $\operatorname{SL}(2, \mathbb{C})$, there is no analogue of the bi-invariant Haar measure dg, which exists on $\mathrm{SU}(2)$. However, thanks to the work of Hall [12], we know there exist unitary holomorphic transforms from the space $L^{2}(\mathrm{SU}(2), \mathrm{d} g)$ of square-integrable functions on $\mathrm{SU}(2)$ to spaces $L^{2}(\mathrm{SL}(2, \mathbb{C}), \mathrm{d} \nu)^{\mathcal{H}}$ of holomorphic and $\nu$-square-integrable functions on $\operatorname{SL}(2, \mathbb{C}$ ). (These are analogous to the Segal-Bargmann integral transform from $L^{2}\left(\mathbb{R}^{n}\right)$ into the holomorphic functions on $\mathbb{C}^{n}$.) This provides us with the desired scalar product on functions on a complex group manifold. Note that Hall's results have recently been used to construct a coherent state transform for spaces of connections in the continuum [13].

We now recall some details of Hall's construction insofar as they are relevant to the present discussion. For each real $t>0$, there is a "coherent-state transform" $C_{t}$ : $L^{2}(\mathrm{SU}(2), \mathrm{d} g) \rightarrow L^{2}\left(\mathrm{SL}(2, \mathbb{C}), \mathrm{d} \nu_{t}\right)^{\mathcal{H}}$ defined by

$$
\left[C_{t}(f)\right]\left(g_{\mathbb{C}}\right):=\int_{S \cup(2)} f(g) \rho_{t}\left(g^{-1} g_{\mathbb{C}}\right) \mathrm{d} g
$$

where $f \in L^{2}(\mathrm{SU}(2), \mathrm{d} g), g \in \mathrm{SU}(2), g_{\mathbb{C}} \in \mathrm{SL}(2, \mathbb{C})$, and $\rho_{t}$ is the heat kernel for the Casimir operator $\Delta=-4 \sum_{i} \hat{p}_{i}^{2}$ on $\mathrm{SU}(2)$, i.e. the fundamental solution at the identity of the heat equation $\mathrm{d} \rho / \mathrm{d} t=\frac{1}{2} \Delta \rho$. More precisely, since the argument of $\rho_{t}$ in (2.9) is a complex group element, we are using its analytic continuation, which is well-defined (see Ref. [12] for details). In terms of the explicit parametrization (2.1) for the matrices $V(g)$ and the normalized Haar measure $\mathrm{d} g$, and using a series expansion for the heat kernel, one obtains

$$
\begin{aligned}
& {\left[C_{t}(f)\right]\left(g_{\mathbb{C}}\right)} \\
& =\frac{1}{\pi^{2}} \int \mathrm{d} \alpha_{0} \int \mathrm{d} \alpha_{1} \int \mathrm{d} \alpha_{2} \int \mathrm{d} \alpha_{3} \delta\left(\sum_{i=0}^{3} a_{i}^{2}-1\right) f\left(\alpha_{i}\right)
\end{aligned}
$$




$$
\begin{aligned}
& \times \sum_{j=0, \frac{1}{2}, \ldots}(2 j+1) \mathrm{e}^{-j(j+1) t / 2} U_{2 j}\left(a_{0} a_{0}^{\mathbb{C}}+a_{1} a_{1}^{\mathbb{C}}+a_{2} a_{2}^{\mathbb{C}}+a_{3} a_{3}^{\mathbb{C}}\right) \\
= & \frac{1}{\pi^{2}} \int_{-1}^{1} \mathrm{~d} \alpha_{0} \int_{-\sqrt{1-a_{0}^{2}}}^{\sqrt{1-a_{0}^{2}}} \mathrm{~d} \alpha_{1} \int_{-\sqrt{1-a_{0}^{2}-a_{1}^{2}}}^{\sqrt{1-a_{0}^{2}-a_{1}^{2}}} \mathrm{~d} \alpha_{2} \frac{1}{2 \sqrt{1-a_{0}^{2}-a_{1}^{2}-a_{2}^{2}}} \sum_{j=0, \frac{1}{2}, \ldots}(2 j+1) \\
& \times \mathrm{e}^{-j(j+1) t / 2}\left(\left.\left(f\left(\alpha_{i}\right) U_{2 j}\right)\right|_{a_{3}=\sqrt{1-a_{0}^{2}-a_{1}^{2}-a_{2}^{2}}}+\left.\left(f\left(\alpha_{i}\right) U_{2 j}\right)\right|_{a_{3}=-\sqrt{1-a_{0}^{2}-a_{1}^{2}-a_{2}^{2}}}\right)
\end{aligned}
$$

(whenever the infinite sum over $j$ on the right-hand side converges), where the $U_{2 j}$ denote the Chebyshev polynomials of the second kind. The image of a square-integrable function $f(g)$ on $\mathrm{SU}(2)$ is a holomorphic function on $\operatorname{SL}(2, \mathbb{C})$, square-integrable with respect to $\mathrm{d} \nu_{t}$, which is essentially the heat kernel measure on the quotient space $\mathrm{SL}(2, \mathbb{C}) / \mathrm{SU}(2)$.

Since our aim is a manifestly gauge-invariant description of lattice gravity in a holomorphic loop representation, several issues have to be resolved. Firstly, we are not aware of a simple explicit expression for the measure $\mathrm{d} \nu_{t}$ in the holomorphic representation obtained via (2.9), and therefore have to look for functions $f(g)$ with a simple transformation behaviour, preferably elements of an orthonormal basis of $L^{2}(\mathrm{SU}(2), \mathrm{d} g$ ), which will be mapped into orthonormal functions of $L^{2}\left(\mathrm{SL}(2, \mathbb{C}), \mathrm{d} \nu_{t}\right)^{\mathcal{H}}$, since $C_{t}$ preserves scalar products. Secondly, we will work with functions that are gauge scalars and can be expressed as functions of Wilson loops, i.e. traced holonomies of closed loops on the lattice. The lattice Hamiltonian maps such functions into themselves.

To obtain the holomorphic transform for general SU(2) wave functions on the lattice, we need to take the product over all lattice links of the transform for a single link, formula (2.10). First however we will give an (overcomplete) set of functions of a single copy of $\mathrm{SU}(2)$, i.e. on a single link, that have a simple transformation behaviour under the transform (2.10). They are given by appropriate sums of polynomials in the four real parameters $\alpha_{i}$ (restricted to the submanifold $\mathrm{SU}(2) \subset \mathbb{R}^{4}$ ) and can be labelled by the exponents $n_{i}$ in the polynomial $\alpha_{0}^{n_{0}} \alpha_{1}^{n_{1}} \alpha_{2}^{n_{2}} \alpha_{3}^{n_{3}}$ of highest order occurring in the sum. One finds

$$
\begin{aligned}
& p\left(n_{0}, n_{1}, n_{2}, n_{3}\right):= \\
& \sum_{j_{0}=1}^{\left\lfloor n_{0} / 2+1 \mid\right.} \frac{(-1)^{j_{0}-1} a_{0}^{n_{0}-2\left(j_{0}-1\right)} \frac{\left(n_{0}\right) !\left(n_{0}-j_{0}+n_{1}+n_{2}+n_{3}+1\right) !}{2^{2\left(j_{0}-1\right)}\left(j_{0}-1\right) !\left(n_{0}-2\left(j_{0}-1\right)\right) !}}{\left(n_{1}+n_{2}+n_{3}\right) !} \\
& \times \sum_{j_{1}=1}^{\left|n_{1} / 2+1\right|} \frac{(-1)^{j_{1}-1} a_{1}^{n_{1}-2\left(j_{1}-1\right)}}{2^{2\left(j_{1}-1\right)}\left(j_{1}-1\right) !\left(n_{1}-2\left(j_{1}-1\right)\right) !} \frac{\left(n_{1}\right) !\left(n_{0}-j_{0}+n_{1}-j_{1}+n_{2}+n_{3}+2\right) !}{\left(n_{0}-j_{0}+n_{1}+n_{2}+n_{3}+1\right) !} \\
& \times \sum_{j_{2}=1}^{\left|m_{2} / 2+1\right|} \frac{(-1)^{j_{2}-1} a_{2}^{n_{2}-2\left(j_{2}-1\right)}}{2^{2\left(j_{2}-1\right)}\left(j_{2}-1\right) !\left(n_{2}-2\left(j_{2}-1\right)\right) !}
\end{aligned}
$$




$$
\begin{gathered}
\times \frac{(n 2) !\left(n_{0}-j_{0}+n_{1}-j_{1}+n_{2}-j_{2}+n_{3}+3\right) !}{\left(n_{0}-j_{0}+n_{1}-j_{1}+n_{2}+n_{3}+2\right) !} \\
\times \sum_{j_{3}=1}^{\left[n_{3} / 2+1\right]} \frac{(-1)^{j_{3}-1} a_{3}^{n_{3}-2\left(j_{3}-1\right)}}{2^{2\left(j_{3}-1\right)}\left(j_{3}-1\right) !\left(n_{3}-2\left(j_{3}-1\right)\right) !} \\
\times \frac{\left(n_{3}\right) !\left(n_{0}-j_{0}+n_{1}-j_{1}+n_{2}-j_{2}+n_{3}-j_{3}+4\right) !}{\left(n_{0}-j_{0}+n_{1}-j_{1}+n_{2}-j_{2}+n_{3}+3\right) !} .
\end{gathered}
$$

For the holomorphic transform of $p\left(n_{0}, n_{1}, n_{2}, n_{3}\right)$ one obtains

$$
\left[C_{t}\left(p\left(n_{0}, n_{1}, n_{2}, n_{3}\right)\right)\right]\left(\alpha_{i}^{\mathbb{C}}\right)=\mathrm{e}^{-\left(n_{0}+n_{1}+n_{2}+n_{3}\right)\left(n_{0}+n_{1}+n_{2}+n_{3}+2\right) t / 8} p\left(n_{0}, n_{1}, n_{2}, n_{3}\right)^{\mathbb{C}}
$$

where by $p\left(n_{0}, n_{1}, n_{2}, n_{3}\right)^{\mathbb{C}}$ we denote the expression (2.11) with the real parameters $\alpha_{i}$ replaced by the corresponding complex quantities $\alpha_{i}^{\mathrm{C}}$. That is, to find the (inverse) holomorphic transform of a polynomial function of the $\alpha_{i}\left(\alpha_{i}^{\mathrm{C}}\right)$, one first has to express it as a linear combination of the $p\left(n_{0}, n_{1}, n_{2}, n_{3}\right)\left(p\left(n_{0}, n_{1}, n_{2}, n_{3}\right)^{\mathbb{C}}\right)$ and then use (2.12). Both (2.11) and (2.12) are crucial formulas for relating the SU(2) and the holomorphic SL $(2, C)$ representation, and will be used in the following sections. The next step is the identification of gauge-invariant combinations of the $p\left(n_{0}, n_{1}, n_{2}, n_{3}\right)$. If the configuration space consisted of just one link (with endpoints identified), our task would be straightforward: all gauge-invariant quantities one can construct in that case are functions of $\frac{1}{2} \operatorname{Tr} V=\alpha_{0}$, and a complete basis is given by the Chebyshev polynomials $U_{n_{0}}\left(\alpha_{0}\right)$.

The situation on the hypercubic lattice is more complicated since general gaugeinvariant quantities are functions of traces of holonomies around arbitrary lattice loops, which do not necessarily factorize into products of link contributions. Moreover, there is the additional problem of finding a set of gauge-invariant functions on $\times_{l} \mathrm{SU}(2)$ that is complete (i.e. spans the Hilbert space of square-integrable functions), but at the same time not overcomplete, i.e. free of redundant degrees of freedom. This is a well-known complication with intrinsically gauge-invariant formulations of lattice gauge theory, and there are various strategies of dealing with it. In Section 4 we will address some of these difficulties in the context of the $1 \times 1 \times 1$ lattice.

Formula (2.11) can be used to construct gauge-invariant functions with a simple transformation behaviour that are labelled by lattice loops. This is important because the (overcomplete) basis of gauge-invariant functions $\left\{\operatorname{Tr} V_{i_{1}} V_{i_{2}} \ldots V_{i_{n}}, \gamma=\right.$ $l_{i_{1}} \circ l_{i_{2}} \circ \ldots \circ l_{i_{n}}$ a lattice loop $\}$ often appears in applications. Using the parametrization (2.1) for the link matrices $V_{i}, \operatorname{Tr} V_{i_{1}} V_{i_{2}} \ldots V_{i_{n}}$ is a homogeneous sum of polynomials in those parameters. Re-expressing in each summand the link contributions in terms of the functions $p\left(n_{0}, n_{1}, n_{2}, n_{3}\right)$, one obtains quantities that transform like (2.12) with $t$-dependent exponential factors. 


\section{Solutions to the Wheeler-DeWitt equation}

Next we study the action of a discretized form of the phase space Hamiltonian for canonical gravity. Let us label lattice sites by an integer $n$ and the three positive directions emanating from each site by $\hat{a}=\hat{1}, \hat{2}$ or $\hat{3}$. Thus the canonical variables are given by $V(n, \hat{a})$ and $p(n, \hat{a})$. We denote the holonomy of a plaquette loop based at the site $n$ in the $\hat{a}-\hat{b}$-plane by $V\left(n, P_{\hat{a} \hat{b}}\right)$, that is, $V\left(n, P_{\hat{a} \hat{b}}\right)=V(n, \hat{a}) V(n+\hat{a}, \hat{b}) V(n+$ $\hat{b}, \hat{a})^{-1} V(n, \hat{b})^{-1}$.

We require that in the limit as all link lengths go to zero the continuum Hamiltonian is recovered. Since our lattice was assumed to be purely topological, we define this limit with respect to an auxiliary Euclidean coordinate system (the three lattice directions coinciding with the three coordinate axes) in which all links have length $a$. As $a \rightarrow 0$, one derives the usual expansion for the plaquette holonomy

$$
V\left(n, P_{\hat{a} \hat{b}}\right)_{A}^{B} \stackrel{a \rightarrow 0}{\longrightarrow} \mathbb{1}_{A}^{B}+a^{2} F_{a b}^{k} \tau_{k A}^{B}+O\left(a^{3}\right),
$$

where $F_{a b}$ is the $a$-b-component of the field strength associated with the selfdual connection $A$. For the momentum variable $p_{i}(n, \hat{a})$ we require that

$$
p_{i}(n, \hat{a}) \stackrel{a \rightarrow 0}{\longrightarrow} a^{2} \tilde{E}_{i}^{a}(n)+O\left(a^{3}\right)
$$

in terms of the continuum momentum density $\tilde{E}$, since $p$ is like a momentum variable smeared in one (out of three) spatial directions. Therefore, if we associate with each lattice site $n$ the lattice Hamiltonian

$$
H^{\mathbb{C}}(n)=\sum_{\hat{a}<\hat{b}} \epsilon^{i j k} p_{i}(n, \hat{a}) p_{j}(n, \hat{b}) \operatorname{Tr}\left(V\left(n, P_{\hat{a} \hat{b}}\right) \tau_{k}\right),
$$

this to lowest order leads, up to a power of $a$, to the correct continuum limit

$$
H^{\mathrm{C}}(n) \stackrel{a \rightarrow 0}{\longrightarrow} a^{6} \epsilon^{i j k} \tilde{E}_{i}^{a} \tilde{E}_{j}^{b} F_{a b k}+O\left(a^{7}\right)
$$

The total Hamiltonian is given by the sum $\sum_{n} H^{\mathrm{C}}(n)$. As a result of the "dimensional transmutation", the discretized Hamiltonian is a topological quantity, in the sense that it depends only on link variables, with no reference to the link length $a$, and acts on loop wave functions in a combinatorial way. As long as we do not re-introduce a length scale, the formulation is therefore purely topological.

Just as in lattice gauge theory, the requirement of the correct continuum limit does not fix the discretized Hamiltonian uniquely. In the present work we do not investigate the question of whether a different choice of $H^{\mathbb{C}}(n)$ leads to equivalent results. In going to the quantum theory, another ambiguity arises in the choice of the operator ordering of $\hat{H}^{\mathbb{C}}(n)$. The most commonly used operator ordering for the Hamiltonian is the one with the operators $\hat{p}_{i}$ to the right, but also the opposite ordering with both of the $\hat{p}_{i}$ to the left is sometimes used [14]. In our present investigation we will be using the former, i.e. 


$$
\hat{H}^{\mathrm{C}}=\sum_{n} \sum_{\hat{a}<\hat{b}} \epsilon^{i j k} \operatorname{Tr}\left(\hat{V}\left(n, P_{\hat{a} \hat{b}}\right) \tau_{k}\right) \hat{p}_{i}(n, \hat{a}) \hat{p}_{j}(n, \hat{b}) .
$$

Since the spatial diffeomorphisms have been taken care of, the only remaining task is to look for holomorphic wave functions $\psi^{\mathbb{C}} \in \times_{l} L^{2}\left(\operatorname{SL}(2, \mathbb{C}), \mathrm{d} \nu_{t}\right)^{\mathcal{H}}$ that solve the analogue of the Wheeler-DeWitt equation,

$$
\hat{H}^{\mathrm{C}} \psi^{\mathbb{C}}=0 \text {. }
$$

The existence of solutions depends on the spectral properties of the Hamiltonian operator $\hat{H}^{\mathbb{C}}$. Since the constituent operators $\hat{V}$ and $\hat{p}$ are not selfadjoint in the holomorphic representation, we do not have any a-priori information about the spectrum of $\hat{H}^{\mathrm{C}}$. There are three possibilities:

(i) $\hat{H}^{\mathbb{C}} \psi^{\mathbb{C}}=0$ has non-trivial solutions in $\times_{l} L^{2}\left(\operatorname{SL}(2, \mathbb{C}), \mathrm{d} \nu_{t}\right)^{\mathcal{H}}$;

(ii) $\hat{H}^{\mathbb{C}} \psi^{\mathbb{C}}=0$ does not have solutions in $\times_{l} L^{2}\left(\operatorname{SL}(2, \mathbb{C}), \mathrm{d} \nu_{t}\right)^{\mathcal{H}}$, but can be solved "as a differential equation", i.e. there are non-square-integrable solutions;

(iii) there are no solutions.

In case (i), the solution space inherits a scalar product from the original Hilbert space, whereas in case (ii) one still has to define a suitable inner product. A number of identities are useful in computing the action of the Hamiltonian on gauge-invariant wave functions (which all contain terms of the form $\operatorname{Tr} V_{i_{1}} V_{i_{2}} \ldots$ ). The first one is

$$
\epsilon^{i j k} \tau_{j A}^{B} \tau_{k C}{ }^{D}=\tau_{i A}{ }^{D} \delta_{C}^{B}-\tau_{i C^{B}} \delta_{A}^{D},
$$

from which follow two identities for the traces of holonomies:

$$
\begin{aligned}
\epsilon^{i j k} \operatorname{Tr}\left(V_{\alpha} \tau_{j} V_{\beta} \tau_{k}\right) & =\operatorname{Tr} V_{\beta} \operatorname{Tr}\left(V_{\alpha} \tau_{i}\right)-\operatorname{Tr} V_{\alpha} \operatorname{Tr}\left(V_{\beta} \tau_{i}\right), \\
\epsilon^{i j k} \operatorname{Tr}\left(V_{\alpha} \tau_{j}\right) \operatorname{Tr}\left(V_{\beta} \tau_{k}\right) & =\operatorname{Tr}\left(V_{\alpha} \tau_{i} V_{\beta}\right)-\operatorname{Tr}\left(V_{\alpha} V_{\beta} \tau_{i}\right),
\end{aligned}
$$

where $\alpha, \beta$ are two lattice loops intersecting at $n$, and the product loop $\alpha \circ \beta$ is obtained by the usual loop composition at $n$. Secondly, we have

$$
\tau_{i A}^{B} \tau_{i C}^{D}=\delta_{A}^{B} \delta_{C}^{D}-2 \delta_{A}^{D} \delta_{B}^{C} .
$$

Lastly, there is the well-known identity relating a product of two Wilson loops of $\mathrm{SL}(2, \mathbb{C})$ holonomies to a sum of two Wilson loops,

$$
\operatorname{Tr} V_{\alpha} \operatorname{Tr} V_{\beta}=\operatorname{Tr} V_{\alpha \circ \beta}+\operatorname{Tr} V_{\alpha \circ \beta^{-1}} \equiv \operatorname{Tr} V_{\alpha} V_{\beta}+\operatorname{Tr} V_{\alpha} V_{\beta}^{-1}
$$

By virtue of these identities, the action of the Hamiltonian on states of the form $\operatorname{Tr} V_{i_{1}} V_{i_{2}} \ldots$ may be interpreted as cutting and joining of the lattice loop arguments, as is typical for the loop representation. To what extent such a geometric interpretation is useful in finding solutions to the zero-eigenvalue equation depends to some extent on the type of basis chosen for the quantum states. As in the case of Hamiltonian lattice gauge theory, the Hamiltonian couples neighbouring links due to the occurrence of the plaquette holonomy operators $\hat{V}\left(n, P_{\hat{a} \hat{b}}\right)$ in $\hat{H}(n)$.

Next we show that solutions to the zero-eigenvalue equation $\hat{H}^{\mathbb{C}} \psi^{\mathbb{C}}=0$ are in oneto-one correspondence with solutions to $\hat{H}_{\text {ind }} \psi^{\mathbb{C}}=0$, where $\hat{H}_{\text {ind }}$ is the (selfadjoint) 
Hamiltonian induced from the $S U(2)$-lattice theory. That is, we take $\hat{H}^{\mathbb{C}}$ as in (3.5), and substitute the operators by their real counterparts acting on $x_{l} L^{2}(S U(2), d g)$ (i.e. take all parameters $\alpha_{i}$ etc. to be real), before translating it to the holomorphic representation using the transform (2.10).

Let us for the moment assume we are given a complete orthogonal basis of wave functions $\left\{\chi(\boldsymbol{n}), \boldsymbol{n}=\left(n_{1}, n_{2}, \ldots n_{d}\right)\right\}, d=\operatorname{dim}\left(\times{ }_{1} \mathrm{SU}(2) / \times_{s} \mathrm{SU}(2)\right)$, for the gaugeinvariant (real) Hilbert space $L^{2}\left(\times_{l} \mathrm{SU}(2) / \times_{s} \mathrm{SU}(2), \pi\left(\prod_{l} \mathrm{~d} g\right)\right.$ ), where $\pi\left(\prod_{l} \mathrm{~d} g\right.$ ) denotes the projection to the quotient space of the product of Haar measures, $l$ is the number of links and $s$ the number of sites. Its elements are labelled by integers $n_{i}$ and transform according to

$$
C_{t}(\chi(\boldsymbol{n}))=\mathrm{e}^{-f_{i}(\boldsymbol{n})} \chi^{\mathbb{C}}(\boldsymbol{n})
$$

and $f_{t}(\boldsymbol{n}) \geqslant 0$. Since the exponential factors are just real numbers rescaling the basis (which we did not assume to be orthonormal), we can write any square-integrable element of the holomorphic Hilbert space as a real linear combination of the $\chi^{\mathbb{C}}(n)$, and have $\left\langle\chi^{\mathbb{C}}(\boldsymbol{m}), \chi^{\mathbb{C}}(\boldsymbol{n})\right\rangle \sim \delta_{m, n}$. The action of the Hamiltonian $\hat{H}^{\mathbb{C}}$ acting on a general vector $\sum_{n} a(\boldsymbol{n}) \chi^{\mathbb{C}}(\boldsymbol{n}), a(\boldsymbol{n}) \in \mathbb{R}$, can be written as a matrix equation

$$
\hat{H}^{\mathbb{C}} \sum_{n} a(n) \chi^{\mathbb{C}}(\boldsymbol{n})=\sum_{n} \sum_{m} M(\boldsymbol{n}, \boldsymbol{m}) a(\boldsymbol{n}) \chi^{\mathbb{C}}(\boldsymbol{m})
$$

The Wheeler-DeWitt equation (3.6) is therefore equivalent to an infinite tower of equations for the coefficients $a(\boldsymbol{n})$,

$$
\sum_{n} M(\boldsymbol{n}, \boldsymbol{m}) a(\boldsymbol{n})=0, \quad \forall \boldsymbol{m}
$$

The norm of a vector $\sum_{n} a(n) \chi^{\mathbb{C}}(n)$ can be calculated using its inverse image under (3.11), and is given by

$$
\| \sum_{n} a(n) \chi^{\mathbb{C}_{(n)} \|_{\operatorname{SL}(2, \mathbb{C})}}=\sqrt{\sum_{n} a(n)^{2} \mathrm{e}^{2 f_{r}(n)}\|\chi(n)\|_{\mathrm{SU}(2)}^{2}} .
$$

By contrast, the Hamiltonian $\hat{H}_{\text {ind }}^{\mathbb{C}}$ acts on holomorphic wave functions according to

$$
\hat{H}_{\text {ind }}^{\mathbb{C}} \sum_{\boldsymbol{n}} b(\boldsymbol{n}) \chi^{\mathbb{C}}(\boldsymbol{n})=\sum_{\boldsymbol{n}} \sum_{\boldsymbol{m}} \mathrm{e}^{-f_{t}(\boldsymbol{m})} M(\boldsymbol{n}, \boldsymbol{m}) \mathrm{e}^{f_{t}(\boldsymbol{n})} b(\boldsymbol{n}) \chi^{\mathbb{C}}(\boldsymbol{m}),
$$

leading to the set of conditions

$$
\sum_{n} M(n, m) \mathrm{e}^{f_{r}(n)} b(n)=0, \quad \forall \boldsymbol{m}
$$

on the coefficients $b(\boldsymbol{n})$ of zero-eigenvectors. Clearly they are related to the solutions of (3.13) by $a(n)=\mathrm{e}^{f_{t}(\boldsymbol{n})} b(\boldsymbol{n})$. However, the norm of the solution vector is in general different, and we have 


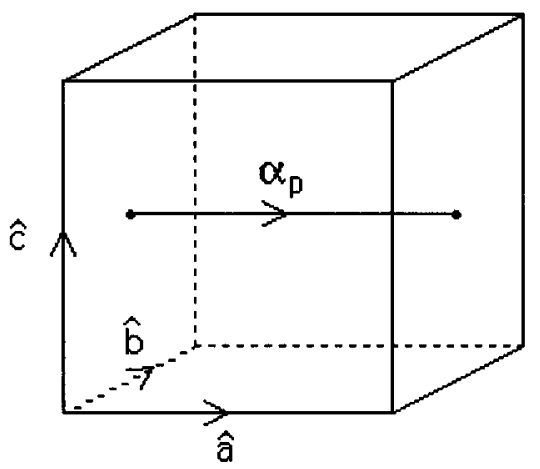

Fig. 1.

$$
\begin{aligned}
\left\|\sum_{n} b(\boldsymbol{n}) \chi^{\mathbb{C}(n)}\right\|_{\mathrm{SL}(2, \mathbb{C})} & =\sqrt{\sum_{n} b(\boldsymbol{n})^{2} \mathrm{e}^{2 f_{1}(\boldsymbol{n})}\|\chi(\boldsymbol{n})\|_{\mathrm{SU}(2)}^{2}} \\
& \leqslant \| \sum_{n} a(\boldsymbol{n}) \chi^{\mathbb{C}(n) \|_{\mathrm{SL}(2, \mathbb{C})}}
\end{aligned}
$$

It may therefore happen that a zero-eigenvalue solution to $H_{\text {ind }}^{\mathrm{C}} \sum_{n} a(\boldsymbol{n}) \chi^{\mathbb{C}}(\boldsymbol{n})=0$ is square-integrable, while the corresponding solution to $\mathscr{H}^{\mathbb{C}} \sum_{n} a(n) \chi^{\mathbb{C}}(n)=0$ is not.

The form of the quantum Hamiltonian (3.5), like its counterpart in the continuum theory, is sufficiently complicated so as not to lead us to expect the eigenvalue problem could be soluble trivially. What comes to help in the analogous problem in the continuum is the existence of "algebraically special" solutions. For example, in the representation where $H_{\text {cont }} \sim \epsilon^{i j k} \tilde{E}_{i}^{a} \tilde{E}_{j}^{b} F_{a b k}$ is quantized by $\hat{H}_{\text {cont }} \sim \epsilon^{i j k} F_{a b k}(A) \partial^{2} / \partial A_{a}^{i} \partial A_{b}^{j}$, solutions are given by wave functions $\psi(A, \gamma):=\operatorname{Tr} P \exp \oint_{\gamma} A_{a}(\gamma(t)) \dot{\gamma}^{a}(t) \mathrm{d} t$, whenever $\gamma$ is a smooth, non-selfintersecting loop [15]. This happens because the derivatives $\partial / \partial A$ bring down two factors of the tangent vector, $\dot{\gamma}^{a} \dot{\gamma}^{b}$, which vanish when multiplied by the antisymmetric tensor $F_{a b}$.

It turns out that there exist analogous solutions in the lattice formulation. Take any straight "Polyakov loop" $\alpha$, i.e. a loop without corners that winds around the lattice once, and is therefore non-contractible. On a $N^{3}$ lattice this is a loop $\alpha$ made up of $N$ consecutive links in a given direction $\hat{a}, \hat{b}$ or $\hat{c}$ (Fig. 1). The contribution of $\hat{H}^{\mathbf{C}}(n)$ to the Hamiltonian acting on a wave function $\sim \operatorname{Tr} V_{\alpha}$ vanishes at any given site $n$ crossed by $\alpha$, since it. would need a wave function with support in at least two independent lattice directions to be non-zero.

There are $3 N^{2}$ such Polyakov loops (which we will take to be positively oriented), $N^{2}$ in each direction. They will be denoted by a subscript $p$. The Polyakov loops can be parametrized by three integers, namely the coordinates of the point where they intersect one of the three planes $\hat{a}=0, \hat{b}=0$ or $\hat{c}=0$. (Note that each lattice link is contained in exactly one Polyakov loop.) We denote the corresponding wave functions by $\phi\left(\alpha_{p}\right) \equiv$ 
$\operatorname{Tr} V_{\alpha_{p}}$. It is easy to see that any wave function $\phi\left(\alpha_{p}^{n}\right)$ of a multiple Polyakov loop $\alpha_{p}^{n}=\alpha \circ \alpha \ldots \circ \alpha$ with winding number $n$ is also annihilated by $\hat{H}^{\mathbb{C}}$. The solution space to the Wheeler-DeWitt equation is therefore infinite-dimensional. However, because of the non-linearity of the Hamiltonian, it is in general not true that $\hat{H}^{\mathbb{C}} \phi\left(\alpha_{p}^{n}\right) \phi\left(\beta_{p}^{m}\right)$ vanishes too. This only occurs when $\left(\alpha_{p}, \beta_{p}\right)$ are a pair of non-intersecting Polyakov loops. We have therefore found: any wave function that is a linear combination of terms in

$$
\left\{\phi\left(\alpha_{p}^{n_{1}}\right) \phi\left(\beta_{p}^{n_{2}}\right) \ldots \phi\left(\omega_{p}^{n_{k}}\right), \quad \alpha_{p}, \beta_{p}, \ldots \omega_{p} \quad k \text { nonintersecting Polyakov loops }\right\}
$$

is annihilated by the discretized Wheeler-DeWitt operator $\hat{H}^{\mathrm{C}}$. The space of such functions is a rather large linear subspace of the original holomorphic Hilbert space. Characteristically, its elements are all highly non-local wave functions on the lattice. Note that we need not consider separately products of the form $\phi\left(\alpha_{p}^{m}\right) \phi\left(\alpha_{p}^{n}\right)$ for multiples of the same Polyakov loop $\alpha_{p}$, since these can always be re-expressed via the trace identity (3.10) as sums of elements of (3.18). Whether there exist solutions that are not of this form remains to be explored ( see also the discussion in the next section).

From the point of view of non-perturbative quantum gravity, one is interested in the structure of the solution space to $H^{\mathbb{C}} \psi^{\mathbb{C}}=0$, in particular its scalar product and natural selfadjoint operators acting on it. For the subsector of Polyakov wave functions described above, there is an induced scalar product from the original Hilbert space of holomorphic wave functions. In deriving this inner product, an ambiguity arises because the scalar product on the wave functions obtained through the holomorphic transform (2.10) is by construction invariant under right and left multiplication by $\mathrm{SU}(2)$ matrices, but not biinvariant under $\operatorname{SL}(2, \mathbb{C})$. However, on the explicitly $(\operatorname{SL}(2, \mathbb{C})$-) gauge-invariant space of Polyakov wave functions there is only a small remnant of this gauge covariance: it turns out that the norm of a complex wave function depends on the number of link variables that appear in the coordinate expression for $\phi\left(\alpha_{p}^{n}\right)$.

This is to be contrasted with the pure SU(2) case, say. There, in order to simplify the computation of scalar products, one often uses a gauge-fixing for a maximal number of link variables, which therefore do not any more appear in the calculation. The right- and left-invariance of the Haar measure ensures that no physical quantities are affected by this choice ( see, for example, Ref. [16]). In the present case of $\operatorname{SL}(2, \mathbb{C})$, one may also introduce a gauge-fixing for some of the links, but one has to keep track of it. Different gauge-fixings result in a rescaling of the wave functions. The norm of a Polyakov wave function $\phi\left(\alpha_{p}\right)$ on a $N^{3}$ lattice without any gauge-fixing can be computed using (2.11), (2.12), and is found to be

$$
\left\|\phi\left(\alpha_{p}\right)\right\|_{\operatorname{SL}(2, \mathbb{C})}=\mathrm{e}^{3 N i / 8} .
$$

If $m$ of the $N$ links occurring in $\alpha_{p}$ are gauge-fixed, the norm changes accordingly to $\mathrm{e}^{3(N-n) t / 8}$. Scalar products between (multiple) Polyakov wave functions $\phi\left(\alpha_{p}^{m}\right)$ and $\phi\left(\beta_{p}^{n}\right)$ vanish whenever the underlying Polyakov loops $\alpha_{p}$ and $\beta_{p}$ are different. 


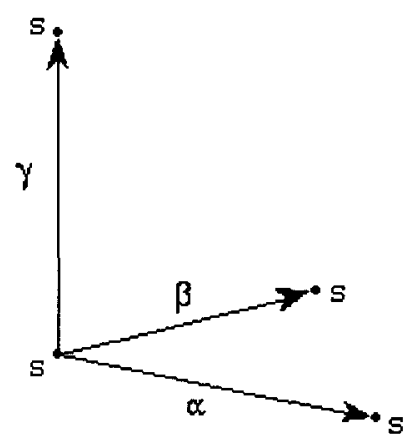

Fig. 2.

Furthermore one finds that for fixed Polyakov loop $\alpha_{p}$, different multiples $\phi\left(\alpha_{p}^{m}\right)$ and $\phi\left(\alpha_{p}^{n}\right)$ generally have a non-zero scalar product, but do not form an orthogonal set, i.e. $\left\langle\phi\left(\alpha_{p}^{m}\right), \phi\left(\alpha_{p}^{n}\right)\right\rangle \neq \delta_{m n}$. Their norms and scalar products can easily be computed using (2.11) and (2.12).

The next step in the investigation is the search for selfadjoint operators, acting on the Hilbert space of the Polyakov wave functions. Natural candidates are the holomorphic transforms of selfadjoint operators in the SU(2) representation that map Polyakov wave functions into themselves. Of course one has to make sure that the final physical expectation values do not depend on a particular gauge-fixing or on the auxiliary parameter $t$. Our lattice formulation does for the first time permit rigorous questions about the physical observables and their operator spectra. A detailed study of these issues will appear elsewhere.

\section{Gravity on the $1 \times 1 \times 1$ lattice}

To illustrate the technicalities involved in the search for solutions other than the Polyakov wave functions of the previous section, we now turn to the case of the $1 \times 1 \times 1$ lattice with periodic boundary conditions. There is a single site $s$ and three (oriented) links emanating from it, which we call $\alpha, \beta$ and $\gamma$ (Fig. 2), to be identified with the $\hat{1}-, \hat{2}$ - and $\hat{3}$-directions on the lattice.

We first discuss the gauge-invariant Hilbert space for the SU(2) theory and then apply the holomorphic transform (2.9), (2.10). By virtue of the boundary conditions, $\alpha, \beta$ and $\gamma$ are themselves closed loops. The corresponding holonomy matrices are parametrized according to $(2.1)$ by real parameters $\alpha_{i}, \beta_{i}$ and $\gamma_{i}$, respectively. The gauge transformations take values in a single copy of the gauge group $\mathrm{SU}(2)$, located at the site $s$. For this case we have complete control over the gauge-invariant functions, i.e. we can give a complete, non-redundant basis of the physical Hilbert space. Following [18], a good set of local coordinates on the six-dimensional physical configuration space 
$\left(\mathrm{SU}(2)_{\alpha} \times \mathrm{SU}(2)_{\beta} \times \mathrm{SU}(2)_{\gamma}\right) / \mathrm{SU}(2)_{s}$ is given by

$$
\begin{aligned}
L_{1}(\alpha) & =\frac{1}{2} \operatorname{Tr} V_{\alpha}=\alpha_{0}, \\
L_{1}(\beta) & =\frac{1}{2} \operatorname{Tr} V_{\beta}=\beta_{0}, \\
L_{1}(\gamma) & =\frac{1}{2} \operatorname{Tr} V_{\gamma}=\gamma_{0}, \\
L_{2}(\alpha, \beta) & =\frac{1}{4}\left(\operatorname{Tr} V_{\alpha} V_{\beta}^{-1}-\operatorname{Tr} V_{\alpha} V_{\beta}\right)=\alpha \cdot \beta, \\
L_{2}(\alpha, \gamma) & =\frac{1}{4}\left(\operatorname{Tr} V_{\alpha} V_{\gamma}^{-1}-\operatorname{Tr} V_{\alpha} V_{\gamma}\right)=\boldsymbol{\alpha} \cdot \boldsymbol{\gamma}, \\
L_{2}(\beta, \gamma) & =\frac{1}{4}\left(\operatorname{Tr} V_{\beta} V_{\gamma}^{-1}-\operatorname{Tr} V_{\beta} V_{\gamma}\right)=\boldsymbol{\beta} \cdot \boldsymbol{\gamma},
\end{aligned}
$$

where we have used a vectorial notation for the "spatial" parameters of the holonomy matrices, $\alpha:=\left(\alpha_{1}, \alpha_{2}, \alpha_{3}\right)$ etc. In order to obtain a good global parametrization, one needs to add a discrete parameter, which we take to be

$$
\operatorname{sign}\left(L_{3}(\alpha, \beta, \gamma)\right)=\operatorname{sign}(\boldsymbol{\alpha} \times \boldsymbol{\beta} \cdot \gamma) .
$$

Also $L_{3}$ may be written as a linear combination of traces of holonomies involving $V_{\alpha}, V_{\beta}$ and $V_{\gamma}$ [17]. The need for such discrete variables has been emphasized by Watson [18]. Roughly speaking, the gauge-invariant Hilbert space of square-integrable functions, $\left.L^{2}\left(\mathrm{SU}(2)_{\alpha} \times \mathrm{SU}(2)_{\beta} \times \mathrm{SU}(2)_{\gamma}\right) / \mathrm{SU}(2)_{s}, \pi\left(\prod_{l} \mathrm{~d} g\right)\right)$ of the quantum theory is spanned by polynomials in the six quantities (4.1) and the discrete variable by a copy of $\mathbb{Z}_{2}$. For making contact with the corresponding $\operatorname{SL}(2, \mathbb{C})$ representation, we have to form a Chebyshev basis from these polynomials, which is orthogonal and has a simple transformation behaviour under (2.10).

For simplicity, we will first describe the subtheory obtained by reduction to the $\hat{\mathbf{1}}-\hat{2}$ plane, say. The relevant classical variables are then $L_{1}(\alpha), L_{1}(\beta)$ and $L_{2}(\alpha, \beta)$. Since the real polynomials $\alpha_{0}^{n_{1}} \beta_{0}^{n_{2}}(\boldsymbol{\alpha} \cdot \boldsymbol{\beta})^{n_{3}}$, regarded as elements of the physical Hilbert space, do not have a straightforward holomorphic transform, we use formula (2.11) to identify appropriate linear combinations of such polynomials, in order to establish the explicit relation between the $\operatorname{SU}(2)$ and the holomorphic $\operatorname{SL}(2, \mathbb{C})$ representations. For those, one finds

$$
\begin{aligned}
& \operatorname{eig}\left(n_{1}, n_{2}, n_{3}\right):=\sum_{j_{0}=1}^{\left[\frac{n_{1}}{2}+1\right]} \frac{(-1)^{j_{0}-1}}{2^{2\left(j_{0}-1\right)}} \frac{n_{1} !\left(n_{1}-j_{0}+n_{3}+1\right) !}{\left(j_{0}-1\right) !\left(n_{1}+n_{3}\right) !\left(n_{1}-2 j_{0}+2\right) !} \\
& \times \sum_{k_{0}=1}^{\left[\frac{m_{2}}{2}+1\right]} \frac{(-1)^{k_{0}-1}}{2^{2\left(k_{0}-1\right)}} \frac{n_{2} !\left(n_{2}-k_{0}+n_{3}+1\right) !}{\left(k_{0}-1\right) !\left(n_{2}+n_{3}\right) !\left(n_{2}-2 k_{0}+2\right) !} \sum_{i=0}^{\left[\frac{n_{3}}{2}\right]} \sum_{k=0}^{i} \sum_{l=0}^{i} \\
& \times\left(\sum_{k_{1}=0}^{k} \sum_{l_{1}=0}^{l}(-1)^{k_{1}}\left(\begin{array}{c}
k \\
k_{1}
\end{array}\right)(-1)^{l_{1}}\left(\begin{array}{c}
l \\
l_{1}
\end{array}\right) a_{0}^{n_{1}-2 j_{0}+2+2 k_{1}} b_{0}^{n_{2}-2 k_{0}+2+2 l_{1}}(\boldsymbol{\alpha} \cdot \boldsymbol{\beta})^{n_{3}-2 i}\right)
\end{aligned}
$$




$$
\begin{aligned}
& \times \prod_{p=0}^{2 i-1}\left(n_{3}-p\right) \prod_{q=0}^{i-l-1} \frac{1}{2\left(n_{2}-k_{0}+n_{3}+1-q\right)} \prod_{r=0}^{i-k-1} \frac{1}{2\left(n_{1}-j_{0}+n_{3}+1-r\right)} \\
& \times\left(\begin{array}{c}
i-k \\
i-k-l
\end{array}\right) \prod_{n=0}^{i-k-l-1}\left(2\left(n_{3}-i-n+\frac{1}{2}\right)(k+n+1)\right) \frac{(-1)^{k+1}}{2^{i}(i-l) !(i-k) !},
\end{aligned}
$$

where $n_{i} \geqslant 0$. To give a few simple examples, one has

$$
\begin{aligned}
& \operatorname{eig}(0,0,0)=1, \\
& \operatorname{eig}(1,0,0)=a_{0}, \\
& \operatorname{eig}(2,0,0)=a_{0}^{2}-\frac{1}{4}, \\
& \operatorname{eig}(0,0,1)=\boldsymbol{a} \cdot \boldsymbol{b}, \\
& \operatorname{eig}(0,0,2)=(\boldsymbol{a} \cdot \boldsymbol{b})^{2}+\frac{a_{0}^{2}}{4}+\frac{b_{0}^{2}}{4}-\frac{5}{16}, \\
& \operatorname{eig}(1,1,1)=a_{0} b_{0} \boldsymbol{a} \cdot \boldsymbol{b}, \\
& \operatorname{eig}(2,1,1)=a_{0}^{2} b_{0} \boldsymbol{a} \cdot \boldsymbol{b}-\boldsymbol{a} \cdot \boldsymbol{b} \frac{b_{0}}{6},
\end{aligned}
$$

Again, by construction, the highest-order polynomial occurring in eig $\left(n_{1}, n_{2}, n_{3}\right)$ is $\alpha_{0}^{n_{1}} \beta_{0}^{n_{2}}(\boldsymbol{\alpha} \cdot \boldsymbol{\beta})^{n_{3}}$, and the remaining lower-order polynomials in the sum ensure a simple transformation behaviour, which in this case is given by

$$
C_{t}\left(\operatorname{eig}\left(n_{1}, n_{2}, n_{3}\right)\right)=\mathrm{e}^{-\left(n_{1}+n_{3}\right)\left(n_{1}+n_{3}+2\right) t / 8} \mathrm{e}^{-\left(n_{2}+n_{3}\right)\left(n_{2}+n_{3}+2\right) t / 8} \operatorname{eig}\left(n_{1}, n_{2}, n_{3}\right) \mathbb{C} .
$$

It is computationally much simpler to determine the action of the Hamiltonian $H^{\mathbb{C}}$ on the non-orthogonal basis of holomorphic, square-integrable wave functions $\left\{e\left(n_{1}, n_{2}, n_{3}\right)^{\mathbb{C}} \equiv\left(\alpha_{0}^{\mathbb{C}}\right)^{n_{1}}\left(\beta_{0}^{\mathbb{C}}\right)^{n_{2}}\left(\boldsymbol{\alpha}^{\mathbb{C}} \cdot \boldsymbol{\beta}^{\mathbb{C}}\right)^{n_{3}}\right\}$, look for zero-eigenvalue solutions and then use $(4.3,5)$ for the computation of scalar products, rather than apply $\hat{H}^{\mathrm{C}}$ on the functions eig $\left(n_{1}, n_{2}, n_{3}\right)^{\mathrm{C}}$ directly. The quantum Hamiltonian for the $1 \times 1 \times 1$ lattice consists of a single contribution $\hat{H}^{\mathbb{C}}(n)$ and is

$$
\hat{H}^{\mathrm{C}}=\sum_{\hat{a}<\hat{b}} \epsilon^{i j k} \operatorname{Tr}\left(\hat{V}\left(P_{\hat{a} \hat{b}}\right) \tau_{k}\right) \hat{p}_{i}(n, \hat{a}) \hat{p}_{j}(n, \hat{b}) .
$$

The plaquette holonomies $V\left(P_{\hat{a} \hat{b}}\right)$ are defined using the appropriate identifications of links, for example, $V\left(P_{\hat{1} \hat{2}}\right)=V_{\hat{1}} V_{\hat{2}} V_{\hat{1}}^{-1} V_{\hat{2}}^{-1}$.

We now describe our method for finding solutions to $\hat{H}^{\mathrm{C}} \sum_{n} m(n) e(n)^{\mathrm{C}}=0$ for the special case that the wave functions have support only in the $\hat{1}-\hat{2}$ plane. We first calculated the action of the Hamiltonian on a basic holomorphic polynomial $e\left(n_{1}, n_{2}, n_{3}\right)^{\mathbb{C}}$, and obtained 


$$
\begin{aligned}
\hat{H}^{\mathbb{C}} e\left(n_{1}, n_{2}, n_{3}\right)^{\mathbb{C}} \\
\quad=n_{1} n_{2}\left(e\left(n_{1}-1, n_{2}-1, n_{3}+3\right)^{\mathbb{C}}-e\left(n_{1}-1, n_{2}-1, n_{3}+1\right)^{\mathbb{C}}\right) \\
+\left(n_{1} n_{2}+n_{1} n_{3}+n_{2} n_{3}-n_{3}\right)\left(e\left(n_{1}, n_{2}, n_{3}\right)^{\mathbb{C}}-e\left(n_{1}, n_{2}, n_{3}+2\right)^{\mathbb{C}}\right) \\
\quad+\left(n_{1} n_{2}-n_{1} n_{3}\right) e\left(n_{1}-1, n_{2}+1, n_{3}+1\right)^{\mathbb{C}} \\
\quad+\left(n_{1} n_{2}-n_{2} n_{3}-\right) e\left(n_{1}+1, n_{2}-1, n_{3}+1\right)^{\mathbb{C}} \\
+\left(n_{1} n_{2}+n_{1} n_{3}+n_{2} n_{3}-n_{3}^{2}\right)\left(e\left(n_{1}+2, n_{2}+2, n_{3}\right)^{\mathbb{C}}\right. \\
\left.\quad-e\left(n_{1}+2, n_{2}, n_{3}\right)^{\mathbb{C}}-e\left(n_{1}, n_{2}+2, n_{3}\right)^{\mathbb{C}}\right) \\
-\left(n_{1} n_{2}-n_{1} n_{3}-n_{2} n_{3}-n_{3}^{2}-2 n_{3}\right) e\left(n_{1}+1, n_{2}+1, n_{3}+1\right)^{\mathbb{C}} \\
+n_{1} n_{3}\left(e\left(n_{1}-1, n_{2}+1, n_{3}-1\right)^{\mathbb{C}}-e\left(n_{1}-1, n_{2}+3, n_{3}-1\right)^{\mathbb{C}}\right) \\
+n_{2} n_{3}\left(e\left(n_{1}+1, n_{2}-1, n_{3}-1\right)^{\mathbb{C}}-e\left(n_{1}+3, n_{2}-1, n_{3}-1\right)^{\mathbb{C}}\right) \\
-\left(2 n_{1} n_{3}+2 n_{2} n_{3}+n_{3}^{2}+2 n_{3}\right) e\left(n_{1}+1, n_{2}+1, n_{3}-1\right)^{\mathbb{C}} \\
+\left(n_{1} n_{3}+2 n_{2} n_{3}+n_{3}^{2}+2 n_{3}\right) e\left(n_{1}+3, n_{2}+1, n_{3}-1\right)^{\mathbb{C}} \\
+\left(2 n_{1} n_{3}+n_{2} n_{3}+n_{3}^{2}+2 n_{3}\right) e\left(n_{1}+1, n_{2}+3, n_{3}-1\right)^{\mathbb{C}} \\
\quad-\left(n_{1} n_{3}+n_{2} n_{3}+n_{3}^{2}+2 n_{3}\right) e\left(n_{1}+3, n_{2}+3, n_{3}-1\right)^{\mathbb{C}} \\
-n_{3}\left(n_{3}-1\right)\left(e\left(n_{1}+2, n_{2}, n_{3}-2\right)^{\mathbb{C}}-e\left(n_{1}+4, n_{2}, n_{3}-2\right)^{\mathbb{C}}\right. \\
+e\left(n_{1}, n_{2}+2, n_{3}-2\right)^{\mathbb{C}}-3 e\left(n_{1}+2, n_{2}+2, n_{3}-2\right)^{\mathbb{C}} \\
+2 e\left(n_{1}+4, n_{2}+2, n_{3}-2\right)^{\mathbb{C}}-e\left(n_{1}, n_{2}+4, n_{3}-2\right)^{\mathbb{C}} \\
\left.+2 e\left(n_{1}+2, n_{2}+4, n_{3}-2\right)^{\mathbb{C}}-e\left(n_{1}+4, n_{2}+4, n_{3}-2\right)^{\mathbb{C}}\right) \\
+
\end{aligned}
$$

for $n_{i} \geqslant 0$. That is, independent of the values of the $n_{i}$, the right-hand side of (4.7) is a linear combination of (at most) 26 terms, and the arguments of the $e\left(n_{1}, n_{2}, n_{3}\right)^{\mathbb{C}}$ occurring lie in the ranges $-1 \leqslant \Delta n_{1} \leqslant 4,-1 \leqslant \Delta n_{2} \leqslant 4$ and $-2 \leqslant \Delta n_{3} \leqslant 3$.

Since the functions $e\left(n_{1}, n_{2}, n_{3}\right)^{\mathbb{C}}$ form a (non-orthogonal) basis for the gaugeinvariant Hilbert space, we can reformulate the zero-eigenvalue condition as an infinite set of equations for the $m\left(n_{1}, n_{2}, n_{3}\right)$ obtained by setting the coefficient of each $e\left(n_{1}, n_{2}, n_{3}\right)^{\mathbb{C}}$ on the right-hand side of $\sum_{n_{i}} \hat{H}^{\mathbb{C}} m\left(n_{1}, n_{2}, n_{3}\right) e\left(n_{1}, n_{2}, n_{3}\right)^{\mathbb{C}}$ to zero. The general condition can be labelled by three integers and is easily derived from (4.7), yielding

$$
\begin{aligned}
C & {\left[n_{1}, n_{2}, n_{3}\right] } \\
& :=\left(n_{1}+1\right)\left(n_{2}+1\right)\left(m\left(n_{1}+1, n_{2}+1, n_{3}-3\right)-m\left(n_{1}+1, n_{2}+1, n_{3}-1\right)\right) \\
& -\left(n_{2} n_{3}-n_{1} n_{2}-n_{1}+n_{3}\right) m\left(n_{1}-1, n_{2}+1, n_{3}-1\right)+\left(n_{1} n_{2}+n_{1} n_{3}+n_{2} n_{3}-n_{3}\right) \\
& \times m\left(n_{1}, n_{2}, n_{3}\right)-\left(\left(n_{1}-2\right) n_{2}+\left(n_{1}-2\right) n_{3}+n_{2} n_{3}-n_{3}^{2}\right) m\left(n_{1}-2, n_{2}, n_{3}\right) \\
& -\left(n_{1} n_{2}+n_{1}\left(n_{3}-2\right)+n_{2}\left(n_{3}-2\right)-n_{3}+2\right) m\left(n_{1}, n_{2}, n_{3}-2\right) \\
& -\left(-n_{1} n_{2}+n_{1} n_{3}-n_{2}+n_{3}\right) m\left(n_{1}+1, n_{2}-1, n_{3}-1\right) \\
& -\left(n_{1}\left(n_{2}-2\right)+n_{1} n_{3}+\left(n_{2}-2\right) n_{3}-n_{3}^{2}\right) m\left(n_{1}, n_{2}-2, n_{3}\right)
\end{aligned}
$$




$$
\begin{aligned}
& -\left(n_{1} n_{2}-n_{1} n_{3}-n_{2} n_{3}-n_{3}^{2}+2 n_{3}\right) m\left(n_{1}-1, n_{2}-1, n_{3}-1\right) \\
& +\left(n_{1}+1\right)\left(n_{3}+1\right)\left(m\left(n_{1}+1, n_{2}-1, n_{3}+1\right)-m\left(n_{1}+1, n_{2}-3, n_{3}+1\right)\right) \\
& +\left(\left(n_{1}-2\right)\left(n_{2}-2\right)+\left(n_{1}-2\right) n_{3}+\left(n_{2}-2\right) n_{3}-n_{3}^{2}\right) m\left(n_{1}-2, n_{2}-2, n_{3}\right) \\
& -\left(2 n_{1} n_{3}+2 n_{2} n_{3}+n_{3}^{2}+2 n_{1}+2 n_{2}-1\right) m\left(n_{1}-1, n_{2}-1, n_{3}+1\right) \\
& +\left(n_{1} n_{3}+2 n_{2} n_{3}+n_{3}^{2}+n_{1}+2 n_{2}-n_{3}-2\right) m\left(n_{1}-3, n_{2}-1, n_{3}+1\right) \\
& +\left(2 n_{1} n_{3}+n_{2} n_{3}+n_{3}^{2}+2 n_{1}+n_{2}-n_{3}-2\right) m\left(n_{1}-1, n_{2}-3, n_{3}+1\right) \\
& -\left(n_{1} n_{3}+n_{2} n_{3}+n_{3}^{2}+n_{1}+n_{2}-2 n_{3}-3\right) m\left(n_{1}-3, n_{2}-3, n_{3}+1\right) \\
& +\left(n_{2}+1\right)\left(n_{3}+1\right)\left(m\left(n_{1}-1, n_{2}+1, n_{3}+1\right)-m\left(n_{1}-3, n_{2}+1, n_{3}+1\right)\right) \\
& -\left(n_{3}^{2}+3 n_{3}+2\right)\left(m\left(n_{1}-2, n_{2}, n_{3}+2\right)-m\left(n_{1}-4, n_{2}, n_{3}+2\right)\right. \\
& +m\left(n_{1}, n_{2}-2, n_{3}+2\right)-3 m\left(n_{1}-2, n_{2}-2, n_{3}+2\right)+2 m\left(n_{1}-4, n_{2}-2, n_{3}+2\right) \\
& \left.-m\left(n_{1}, n_{2}-4, n_{3}+2\right)+2 m\left(n_{1}-2, n_{2}-4, n_{3}+2\right)-m\left(n_{1}-4, n_{2}-4, n_{3}+2\right)\right) \\
& =0 .
\end{aligned}
$$

Notice first that the two-dimensional wave functions $e\left(n_{1}, n_{2}, n_{3}\right)^{\mathbb{C}}$ fall into two sectors which are mapped into themselves under the action of the Hamiltonian. These are (i) the even sector: either all $n_{i}$ are even or all $n_{i}$ are odd; (ii) the odd sector: two $n_{i}$ are even and one $n_{i}$ is odd or vice versa. Hence it suffices to investigate the two sectors separately.

The special solutions discussed in the previous section correspond to all functions of the form $e\left(n_{1}, 0,0\right)^{\mathbb{C}}$ and $e\left(0, n_{2}, 0\right)^{\mathbb{C}}$, and (4.8) imposes no conditions on the corresponding coefficients $m\left(n_{1}, 0,0\right)$ and $m\left(0, n_{2}, 0\right)$. We will in the following set these coefficients to zero, because we are interested in the possible existence of other solutions. In order to tackle the system of equations in a well-defined manner, it is useful to define the order ord $\left(C\left[n_{1}, n_{2}, n_{3}\right]\right)$ by

$$
\operatorname{ord}\left(C\left[n_{1}, n_{2}, n_{3}\right]\right):=\max \left\{n_{1}+n_{3}, n_{2}+n_{3}\right\}
$$

and then try to solve simultaneously, order by order, the sets of Eqs. (4.8) of the same order, eliminating coefficients $m\left(n_{1}, n_{2}, n_{3}\right)$ of higher order (with 'order' defined analogously to (4.9)) in terms of the lower order ones. We have investigated the even sector and solved iteratively in this manner the order- $0,2,4,6,8,10$ and 12 equations. There are 1 order- 0 equation, 5 order $-2,13$ order- 4,25 order- 6,41 order-8, 61 order-10 and 85 order-12 equations. Taking into account equations up to this order, one finds that for any solution to the Wheeler-DeWitt equation the coefficients have to satisfy simultaneously 


$$
\begin{aligned}
\text { order } 2: m(0,0,2) & =m(1,1,1)=m(2,2,0)=0 \\
\text { order 4: } m(1,3,1) & =m(3,1,1)=m(1,1,3)=m(2,0,2) \\
& =m(0,2,2)=m(0,0,4)=0 \\
m(2,4,0) & =m(4,2,0)=\frac{1}{9} m(2,2,2), \quad m(3,3,1)=\frac{38}{27} m(2,2,2), \\
& \text { no conditions on } m(2,2,2), \quad m(4,4,0) \\
\text { order 6: } m(1,5,1) & =m(5,1,1)=-\frac{7}{9} m(2,2,2), \quad m(1,1,5)=\frac{2}{45} m(2,2,2), \\
m(4,0,2) & =m(0,4,2)=-\frac{7}{18} m(2,2,2), \\
m(3,1,3) & =m(1,3,3)=\frac{13}{27} m(2,2,2), \\
m(0,0,6) & =-\frac{1}{135} m(2,2,2), \ldots,
\end{aligned}
$$

i.e. the coefficients $m$ of order 0 and 2 are completely fixed, but at order 4 there appear two free parameters, $m(2,2,2)$ and $m(4,4,0)$. (Of course we could have solved in terms of other order-4 parameters.) Unfortunately we cannot be sure whether the conditions $C\left[n_{1}, n_{2}, n_{3}\right]=0$ for $\operatorname{ord}(C)>12$ do not (through coupling among equations of different order) fix these parameters, although the behaviour of the conditions evaluated so far does not make it seem likely. We conjecture that there is an infinite number of free parameters (with an increasing number of free parameters at each order), corresponding to an infinite set of solutions to the discretized Wheeler-DeWitt equation (beyond those coming from the Polyakov loops). These would be of the form of (presumably infinite) linear combinations, parametrized by those free parameters. Clearly then the question arises of whether these solutions have a finite norm. Since we have not even found a single explicit solution of this type, we are unable to answer this question presently.

We will now have a brief look at the full $1 \times 1 \times 1$ lattice theory. However, we will not attempt to solve the eigenvalue equation directly, since the analogues of (4.7) and (4.8) contain about ten times as many terms. The discussion is meant to serve as an illustration of how to set up the gauge-invariant Hilbert space in a symmetric way, and formulate the eigenvalue problem in principle. The problem is the incorporation of the classical discrete degree of freedom $\operatorname{sign}\left(L_{3}(\alpha, \beta, \gamma)\right)$ in the quantum theory. Although the functions

$$
\left\{\alpha_{0}^{n_{1}} \beta_{0}^{n_{2}} \gamma_{0}^{n_{3}}(\boldsymbol{\alpha} \cdot \boldsymbol{\beta})^{n_{4}}(\boldsymbol{\alpha} \cdot \boldsymbol{\gamma})^{n_{5}}(\boldsymbol{\beta} \cdot \boldsymbol{\gamma})^{n_{6}}(\operatorname{sign}(\boldsymbol{\alpha} \times \boldsymbol{\beta} \cdot \boldsymbol{\gamma}))^{z}, n_{i}=0,1,2, \ldots, z=0,1\right\}
$$

could in principle serve as a basis for the gauge-invariant Hilbert space, it is difficult to incorporate $\operatorname{sign}(\boldsymbol{\alpha} \times \boldsymbol{\beta} \cdot \boldsymbol{\gamma})$ in integrations, since it is not a smooth function on the classical group manifold. Another admissible choice is

$$
\left\{\alpha_{0}^{n_{1}} \beta_{0}^{n_{2}} \gamma_{0}^{n_{3}}(\boldsymbol{\alpha} \cdot \boldsymbol{\beta})^{n_{4}}(\boldsymbol{\alpha} \cdot \boldsymbol{\gamma})^{n_{5}}(\boldsymbol{\alpha} \times \boldsymbol{\beta} \cdot \boldsymbol{\gamma})^{n_{6}}, n_{i}=0,1,2, \ldots\right\},
$$

but this is not symmetric with respect to the three lattice directions. A choice that solves both of these problems is 


$$
\begin{aligned}
\left\{e\left(n_{1}, n_{2}, n_{3}, n_{4}, n_{5}, n_{6}, z\right):=\right. & \alpha_{0}^{n_{1}} \beta_{0}^{n_{2}} \gamma_{0}^{n_{3}}(\boldsymbol{\alpha} \cdot \boldsymbol{\beta})^{n_{4}}(\boldsymbol{\alpha} \cdot \boldsymbol{\gamma})^{n_{5}}(\boldsymbol{\beta} \cdot \boldsymbol{\gamma})^{n_{6}}(\boldsymbol{\alpha} \times \boldsymbol{\beta} \cdot \boldsymbol{\gamma})^{z}, \\
& \left.n_{i}=0,1,2, \ldots, z=0,1\right\} .
\end{aligned}
$$

It contains all gauge-invariant information about the original Hilbert space, without being overcomplete. There is a corresponding, non-orthogonal basis for the squareintegrable, holomorphic wave functions, obtained by substituting as usual the real parameters in (4.13) by their complex counterparts. Acting with the Hamiltonian $\hat{H}^{\mathrm{C}}$ on such a state may produce terms that contain higher-order powers $\left(\boldsymbol{\alpha}^{\mathbb{C}} \times \boldsymbol{\beta}^{\mathbb{C}} \cdot \boldsymbol{\gamma}^{\mathbb{C}}\right)^{n}, n>1$, which then have to be re-expressed as a sum of (complex) terms of the form (4.13) using the identity

$$
\begin{aligned}
\left(\boldsymbol{\alpha}^{\mathbb{C}} \times \boldsymbol{\beta}^{\mathbb{C}} \cdot \boldsymbol{\gamma}^{\mathbb{C}}\right)^{2}= & \left(1-\left(\boldsymbol{\alpha}^{\mathbb{C}}\right)^{2}\right)\left(1-\left(\boldsymbol{\beta}^{\mathbb{C}}\right)^{2}\right)\left(1-\left(\boldsymbol{\gamma}^{\mathbb{C}}\right)^{2}\right)-\left(1-\left(\boldsymbol{\alpha}^{\mathbb{C}}\right)^{2}\right)\left(\boldsymbol{\beta}^{\mathbb{C}} \cdot \boldsymbol{\gamma}^{\mathbb{C}}\right)^{2} \\
& -\left(1-\left(\boldsymbol{\beta}^{\mathbb{C}}\right)^{2}\right)\left(\boldsymbol{\alpha}^{\mathbb{C}} \cdot \boldsymbol{\gamma}^{\mathbb{C}}\right)^{2}-\left(1-\left(\boldsymbol{\gamma}^{\mathbb{C}}\right)^{2}\right)\left(\boldsymbol{\alpha}^{\mathbb{C}} \cdot \boldsymbol{\beta}^{\mathbb{C}}\right)^{2} \\
& +2\left(\boldsymbol{\alpha}^{\mathbb{C}} \cdot \boldsymbol{\beta}^{\mathbb{C}}\right)\left(\boldsymbol{\alpha}^{\mathbb{C}} \cdot \boldsymbol{\gamma}^{\mathbb{C}}\right)\left(\boldsymbol{\beta}^{\mathbb{C}} \cdot \boldsymbol{\gamma}^{\mathbb{C}}\right) .
\end{aligned}
$$

This is an unambiguous prescription and leads to a Hamiltonian action that maps states of the form $\sum m\left(n_{1}, n_{2}, n_{3}, n_{4}, n_{5}, n_{6}, z\right) e\left(n_{1}, n_{2}, n_{3}, n_{4}, n_{5}, n_{6}, z\right) C$ into themselves. However, as already mentioned, the Hamiltonian is a lengthy expression and solving the zero-eigenvalue problem is certainly not a straightforward task. This is probably just a reflection of the non-triviality of quantum gravity. Still we cannot rule out the existence of a basis for the gauge-invariant Hilbert space that leads to a simplification of the eigenvalue problem. However, this would presumably be an overcomplete basis, which then leads to problems of a different kind. Firstly, one cannot just set the coefficients of each "basis" wave function to zero, and secondly one has to eliminate spurious solutions by hand. By contrast, an advantage of our formulation is that the general condition on the wave function coefficients $m(n)$ is known explicitly, so that one has full control over all the relevant physical parameters. This is important if one for instance decides to introduce a cut-off in the Hilbert space, in order to approximate the problem of finding zero-eigenvectors by a finite-dimensional one.

\section{Conclusions}

We have described above a regularized version of non-perturbative canonical quantum gravity on a cubic lattice with periodic boundary conditions. The quantum Hamiltonian on the lattice acts combinatorially on holomorphic wave functions labelled by lattice loops. The lattice represents an entire diffeomorphism equivalence class and the formalism is manifestly gauge- and diffeomorphism-invariant at the kinematical level. With a specific choice for the discretized Hamiltonian and a factor ordering in the quantum theory, we are able to identify an infinite-dimensional space of solutions to the discretized Wheeler-DeWitt equation, which moreover have finite norm with respect to a natural scalar product on the space of holomorphic $\operatorname{SL}(2, \mathbb{C})$ functions. The solution space is labelled by global Polyakov loops and their multiples, and corresponds to $\sim 3 N^{2}$ physical 
degrees of freedom (compared to $\sim 6 N^{3}$ before imposing the Hamiltonian constraint). Still, more research is needed to determine whether this exhausts the solution space. For the example of the $1 \times 1 \times 1$ lattice, we illustrated how one may go about a systematic search for more solutions. Although we have not yet been able to find any, preliminary results suggest there may be an infinite set of solutions beyond the Polyakov ones. However, even if that is the case, it may still happen that they are not square-integrable.

Since our lattice regularization is rather different from the point-split regularization used in the formal continuum approaches, it is remarkable that our solution space is reminiscent of the smooth, non-intersecting solutions of Ref. [6] (thus suggesting that these in fact are not "spurious"). We expect this feature to be fairly robust under a change of the classical discretized Hamiltonian, because the existence of the solutions depends only on the antisymmetric structure of $\epsilon^{i j k} p_{j} p_{k}$. However, we have not found straightforward analogues of the intersecting-loop solutions of Refs. [15,19]; this can be traced back to the non-locality of the lattice Hamiltonian. The great advantage of our regularization is the existence of a well-defined scalar product at every stage. This will be crucial in all further investigations of the solution space. For example, it would be interesting to understand how a different factor ordering of $\hat{H}^{\mathbb{C}}$ changes our results.

In order to avoid confusion, it should be pointed out that we are proceeding somewhat differently from the path outlined (for the continuum theory) by Ashtekar and collaborators $[20,13]$. They propose to solve the gauge and diffeomorphism constraints within a real framework, based on the spin connection $\Gamma_{a}^{i}$, and then go to a holomorphic representation to solve the Hamiltonian constraint, whereas our formulation takes place entirely within the complex formulation based on $A_{a}^{i}=\Gamma_{a}^{i}-i K_{a}^{i}$ (where $K_{a}^{i}$ is related to the extrinsic curvature via $K_{a}^{i}=K_{a b} E^{b i}$ ). This does not exclude that a close relation may emerge at the level of dynamics.

Finally, as already mentioned in the introduction, one has to face the question of the continuum limit (not to be confused with a weak-field limit) of the regularized theory. The diffeomorphism invariance of general relativity makes this a fundamentally different issue from that in lattice gauge theory. If one wants to avoid bringing back in an ultraviolet cutoff $a$, the only free parameter is the lattice size $N$, and one would expect that in the limit for growing $N$ a prospective continuum theory is approximated ever better. Since (at least part of) the solution space is known for every finite $N$ (and the $N$-dependence of our construction is rather explicit), one might investigate the limit as $N \rightarrow \infty$ of these spaces directly. This will probably become more meaningful once observables and possibly matter fields have been included, so that one can study their spectral properties as a function of $N$. Our construction suggests that in such a "continuum" limit some fundamental discrete structure is retained, although (as pointed out in Ref. [21]) this does not necessarily preclude the appearance of divergences. 


\section{Acknowledgements}

The author was supported by a grant under the European Human Capital and Mobility program on "Constrained Dynamical Systems".

\section{References}

[1] J. Jurkiewicz, Simplicial gravity and random surfaces, Nucl. Phys. B Proc. Suppl. 30 ( 1993 ) 108.

[2] P. Renteln, Some results of SU(2) spinorial lattice gravity, Class. Quant. Grav. 7 (1990) 493.

[3] T. Piran and R. Williams, Phys. Rev. D 33 (1986) 1622;

J.L. Friedman and I. Jack, J. Math. Phys. 27 (1986) 2973;

P. Renteln and L. Smolin, Class. Quant. Grav. 6 (1989) 275;

O. Boström, M. Miller and L. Smolin, A new discretization of classical and quantum general relativity, preprint Göteborg U. ITP 94-5 and Syracuse U. SU-GP-93-4-1.

[4] A. Ashtekar, Phys. Rev. Lett. 57 (1986) 2244; Phys. Rev. D36 (1987) 1587.

[5] A. Ashtekar, Lectures on non-perturbative canonical gravity (World Scientific, Singapore, 1991).

[6] C. Rovelli and L. Smolin, Nucl. Phys. B 331 (1990) 80.

17] B. Brügmann, Loop representations, in Canonical gravity: from classical to quantum, ed. J. Ehlers and H. Friedrich, Lecture Notes in Physics 434 (Springer, Berlin, 1994).

[8] A. Ashtekar and J. Lewandowski, Projective techniques and functional integration for gauge theories, to appear in J. Math. Phys. special issue on Functional Integration, ed. C. DeWitt-Morette, e-Print Archive: gr-qc 9411046.

[9] K.V. Kuchař, Phys. Rev. D 34 (1986) 3044; see also C.J. Isham: Quantum gravity, in General relativity and gravitation, Proc. 11th Int. Conf. ed. M.A.H. MacCallum (Cambridge University Press, Cambridge, 1987).

[10] L.D. McLerran and B. Svetitsky, Phys. Lett. B 98 (1981) 195;

J. Kuti, J. Polónyi and K. Szlachányi, Phys. Lett. B 98 (1981) 199.

[11] J. Kogut and L. Susskind, Phys. Rev. D 11 (1975) 395;

J.B. Kogut, Rev. Mod. Phys, 55 (1983) 775.

[12] B.C. Hall, J. Funct. Anal. 122 (1994) 103.

[13] A. Ashtekar, L. Lewandowski, D. Marolf, J. Mourão T. Thiemann, Coherent state transform for spaces of connections, preprint Penn State U., Dec 1994, e-Print Archive: gr-qc 9412014.

[14] B. Brügmann, R. Gambini and J. Pullin, Nucl. Phys. B 385 (1992) 587.

[15] T. Jacobson and L. Smolin, Nucl. Phys. B 299 (1988) 295.

[16] M. Creutz, Quarks, gluons and lattices (Cambridge University Press, Cambridge, 1983).

[17] R. Loll, Nucl. Phys. B 368 (1992) 121; B 400 (1993) 126.

[18] N.J. Watson, Gauge invariant variables and Mandelstam constraints in SU(2) gauge theory, preprint Marseille CPT-94-P-3065, Aug 1994, e-Print Archive: hep-th 9408174

[19] V. Husain, Nucl. Phys. B 313 (1989) 711; B. Brügmann and J. Pullin, Nucl. Phys. B 363 (1991) 221.

[20] A. Ashtekar, Recent mathematical developments in quantum general relativity, to appear in Proceedings of the VIIth Marcel Grossmann Conference, ed. R. Ruffini and M. Keiser (World Scientific, Singapore, 1995) e-Print Archive: gr-qc 9411055.

121] C. Rovelli and L. Smolin, Nucl. Phys. B 442 (1995) 593. 